\title{
Wideband Transimpedance Amplifiers for Optoelectronics: Applications to Dynamic Interferometry
}

\author{
Lucas M. Riobó ${ }^{1 * \dagger}$, Francisco E. Veiras ${ }^{* \dagger}$,María T. Garea* and Patricio A. Sorichetti* \\ *Universidad de Buenos Aires. Facultad de Ingeniería. Departamento de Física. GLOmAe. \\ Av. Paseo Colón 850, CABA C1063ACV, Argentina \\ ${ }^{\dagger}$ CONICET \\ Godoy Cruz 2290 (C1425FQB) CABA - República Argentina \\ ${ }^{1}$ Irioboefi.uba.ar \\ Recibido: 23/06/17; Aceptado:14/07/17
}

\begin{abstract}
This paper describes the design and performance of transimpedance amplifiers (TIAs) for optoelectronic systems, optimized for optical dynamic interferometry. In order to perform the analysis of the amplifiers, we show some experimental schemes, where different topologies were used. We describe the relevance of each TIA implemented and the corresponding guidelines and design considerations.
\end{abstract}

Resumen- Este artículo describe el diseño y desempeño de amplificadores de transimpedancia (TIAs) para optoelectrónica, optimizados para su uso en aplicaciones de interferometría óptica dinámica. Para desarrollar el análisis de los amplificadores, mostramos algunos esquemas experimentales donde se utilizan distintas topologías. En cada esquema desarrollado, describiremos la relevancia de cada TIA, mencionando lineamientos y consideraciones de diseño.

\section{INTRODUCTION: DETECTION OF OPTICAL INTERFEROMETRIC SIGNALS}

In optical sensing systems, the current generated from photodetectors is generally small. Moreover, most of the subsequent processing occurs in the voltage domain. Thus, a conversion from current to voltage is required. A current to voltage converter is also called a transimpedance amplifier (TIA). TIAs are commonly used in many applications that require high dynamic range, speed and sensivity, such as optical communications and optical metrology systems [1].

It is known that optical interferometric methods allow the measurement of sub-nanometer dynamic displacements [2], [3]. These techniques have been applied to a variety of determinations [4], [5] and are particularly interesting in the case of electromechanical properties of materials [6], single nanoparticle detection in fluids [7] or observation of gravitational waves [8]. Since the light used for sensing usually does not alter the sample under study, optical interferometric techniques also present significant advantages for non-destructive testing (NDT) [9].

A general expression of a two-beam interferometric signal is shown on (14):

$$
I(\vec{r}, t)=A(\vec{r}, t)+B(\vec{r}, t) \cos \theta(\vec{r}, t)
$$

where $A(\vec{r}, t)$ is the background intensity, $B(\vec{r}, t)$ is the fringe contrast and $\theta(\vec{r}, t)$ is the phase difference between the interfering waves. These parameters can be both space $(\vec{r})$ and time $(t)$ dependent. Usually, the information of interest is encoded on $\theta(\vec{r}, t)$. Thus, we need to detect and process $I(\vec{r}, t)$ without any kind of distortion.

An interferometry system can be divided in three functional blocks. The first block is the experimental scheme on which the device under test (DUT) is placed and the interfering beams are generated and combined. The second block is the detection block whose front-end can be either a camera or single photodetectors, or both. The third block is the processing block that is used to extract the required information.

Due to the limited capturing rate of the camera, initially camera-based interferometers were mainly used for quasi static measurements. For high-frequency vibration measurement, there are various techniques that allow steady-state surface vibration mode measurements, but these can only be applied in principle on periodical disturbances [10]. Non periodical measurements can be made by high speed cameras, having frame rates in excess of 250 frames per second. However, they can be very expensive in comparison to high speed single photodetectors. Moreover, they may not be capable of working with low intensity signals. In addition, if low-speed CCDs are used, the time for acquiring the interferograms may be quite long and this makes the technique poorly suited to be used in an industrial environment [11].

In this work, we are interested in the detection of time varying interferometric signals. For this purpose we use photodiodes as photodetectors. A photodiode converts the photon energy of light into an electrical signal by releasing and accelerating current-conducting carriers within the semiconductor. In this case, the diode junction is customized improving their spectral response and efficiency as in PIN and avalanche alternatives Also, multiple-element and lateral photodiodes provide position-sensing through the relative magnitudes of multiple output currents. By itself, a photodiode can produce a voltage output as required for most electronic instrumentation. However, this operating mode (photovoltaic mode) produces a highly nonlinear response and a very restricted bandwidth. Instead, accepting the diode output as a current (photoconductive mode) and performing a current-to-voltage conversion dramatically improves its performance [1].

In the following sections, we present three different interferometric schemes, each of them using a specific detection 
setup. The first setup is the simplest form of current to voltage conversion. We take advantage of its simplicity to study the detection process of photodiodes and also to define our design considerations in optoelectronic measurements. In the second setup we present a photodiode transimpedance amplifier. We describe the critical parameters involved in the design of a transimpedance amplifier destined to measure interferometric signals with low modulation depth. The last setup is a wideband photodiode transimpedance amplifier which can detect high speed optical signals. Finally, the results of this work are discussed.

\section{FIRST APPROACH: DETECTION OF LOW SPEED PHENOMENA}

Consider the birefringent phase demodulator shown in Fig.1, detailed in [12].

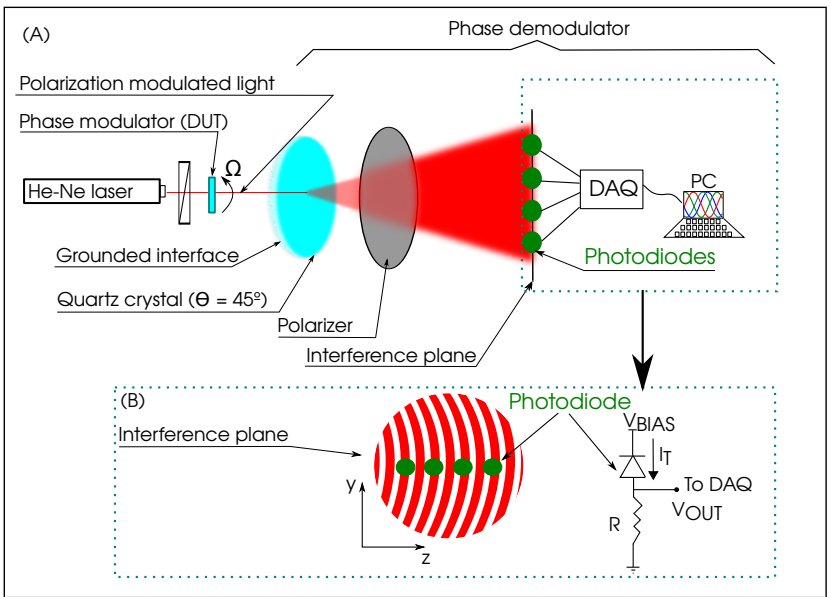

Fig. 1. Birefringent phase demodulator. (A) An array of photodiodes is placed in the interference plane. The photocurrent $I_{T}$ is converted to a voltage signal $\left(V_{O U T}\right)$ by a resistor $R$ and then sampled by a data acquisition system (DAQ) and processed by a computer (PC) [13]. (B) Distribution of the photodiodes and connection scheme.

A spatiotemporal interferogram is produced by the system itself and detected by an array of photodiodes. Each photodiode is placed in a position $\overrightarrow{r_{i}}=\left(y_{i}, z_{i}\right)$ where the intensity is

$$
I\left(\overrightarrow{r_{i}}, t\right)=A\left(\overrightarrow{r_{i}}\right)+B\left(\overrightarrow{r_{i}}\right) \cos \left[\Delta \phi_{o-e}\left(\overrightarrow{r_{i}}\right)+\Delta \phi_{v-h}(t)\right]
$$

Here $\Delta \phi_{o-e}\left(\vec{r}_{i}\right)$ is a fixed spatial phase and $\Delta \phi_{v-h}(t)$ is the phase produced by the device under test. This phase can be recovered by applying

$$
\widehat{\Delta} \phi_{v-h}(t)=\arctan \left[\frac{\sum_{i=1}^{N} n_{i} \tilde{I}\left(\overrightarrow{r_{i}}, t\right)}{\sum_{i=1}^{N} d_{i} \tilde{I}\left(\overrightarrow{r_{i}}, t\right)}\right]
$$

where $\widehat{\Delta} \phi_{v-h}(t)$ is the estimated phase and $N$ is the total number of photodetectors. Each intensity is converted into a photocurrent and then converted again into a voltage signal by a resistor $R$. Both weighted summations of photodiode signals in the numerator and denominator can be performed electronically. Therefore, in a post processing stage, the arctangent can be calculated.

The interferogram provided by the system in Fig.1 can be very diffuse, depending on the optical components used. In addition, the interferogram has a large background intensity.
The active surface area of the photodiodes plays a key role on the performance of the detector. If it is very small, the effect of speckle in the interferogram will decrease the signal to noise ratio (SNR) of the detected signal. If the area of the photodiode is too large, the SNR will also decay because we lose spatial resolution [13]. Since the position of the photodiodes can be adjusted, we can use large area detectors in order to measure the intensity signals (Fig. 1) without saturation and loss of spatial resolution in expense of signal strength. Therefore, the current to voltage converter needs to be sensitive to the intensity variations produced by $\Delta \phi_{v-h}(t)$ under low spatial modulation depth.

\section{A. Characteristics of the current to voltage converter}

As we can see in (3), to effectively retrieve $\Delta \phi_{v-h}(t)$, the current to voltage conversion needs to be linear. The photodiode response is linear when we sample the photocurrent under the conditions $V_{B I A S} \geq 0$ and $V_{B I A S}>$ $V_{O U T}=I_{T} R$ (Fig.1 (B)). The total current provided by the photodiode is $I_{T}=I_{P H}+I_{\text {Dark }}$, where $I_{P H}$ is the photocurrent and $I_{\text {Dark }}$ is the dark current. The dark current is the current that will flow through the photodiode under nolight conditions and it increases with $V_{B I A S}$. The bandwidth $(B W)$ of the system is determined by the sample resistor $R$ and the junction capacitance of the photodiode $C_{P H}$, which decreases with $V_{B I A S}: B W=1 /\left(2 \pi R C_{P H}\right)$. The noise sources are mainly: shot noise in the photodiodes, thermal noise in the sample resistor $R$, and external coupled interfering signals. A complete study of different noise contributions on the current to voltage converter is detailed in [14].

In our experimental setup we used four photodiodes Vishay BPW43 [15] reverse biased at $9 \mathrm{~V}$ with a battery, each with a sample resistor $R=1 \mathrm{M} \Omega$. The measured SNR is approximately $80 \mathrm{~dB}$ on a $10 \mathrm{kHz}$ bandwidth. A picture of the detector is shown on Fig.2.

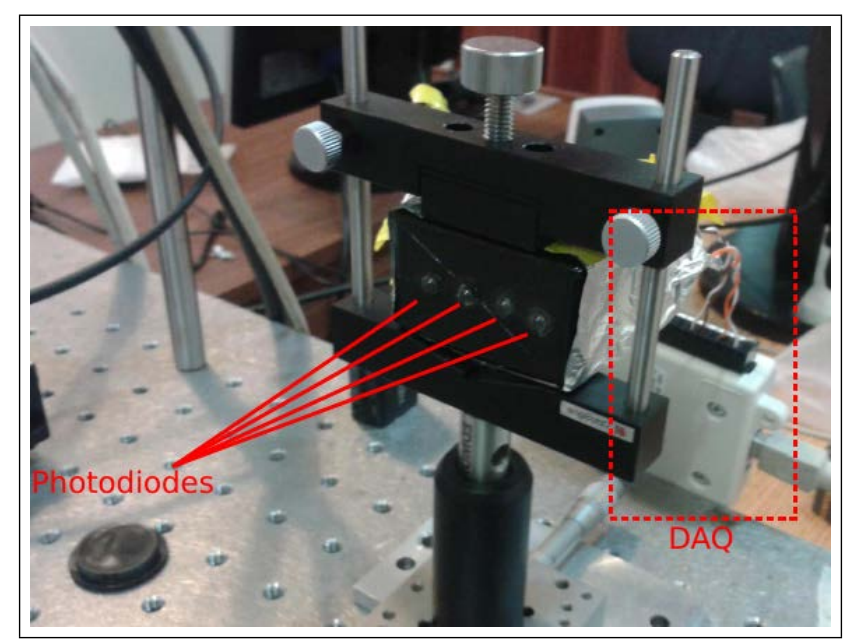

Fig. 2. Detector implemented. The photodiodes are placed into a metal box to prevent external noise sources from entering the system. [13]

This current to voltage converter has the advantage of being simple to construct and understand. The main limitation of this configuration is the bandwidth, which turns out to be a critical issue when detecting low intensity signals. The bandwidth can be increased by means of a reduction of the 
value of $R$ at the expense of reducing the SNR. Another way of widening the bandwidth is by increasing the reverse bias of the photodiodes. However, this increases the dark current and consequently reduces the SNR of the detector. In the next section, we analyze the transimpedance amplifier (TIA) based on operational amplifiers. The TIA is the topology most frequently used to detect optical signals. Compared to the current to voltage converter previously described, it improves the linearity of the photodiode, the SNR and the bandwidth. However, the analysis of the TIA is far more complex: the inclusion of an active element and a feedback network requires careful attention to stability issues and noise contributors of the operational amplifier.

\section{SECOND APPROACH: DETECTION OF HIGH SPEED PHENOMENA}

Consider the experimental setup of Fig. 3. The detailed description of the system and the operation principles are in [6].

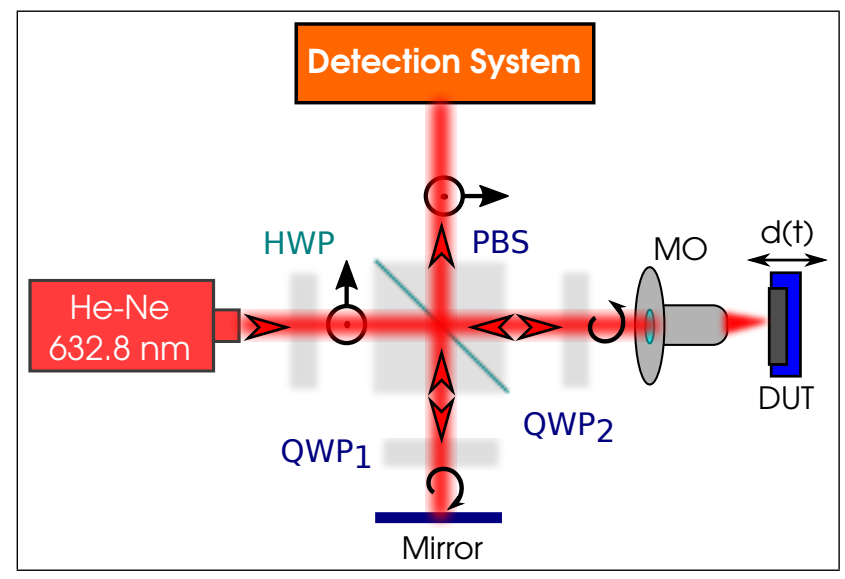

Fig. 3. A Polarization Michelson interferometer. HWP: half waveplate PBS: polarized beam splitter, $\mathrm{QWP}_{1,2}$ : quarter waveplates, $\mathrm{MO}$ : microscope objective. $d(t)$ is the off-plane displacement of the Device Under Test (DUT) to be measured. [6]

The physical displacement of the DUT modulates the polarization state of the light reaching the detector. If there is a single polarizer in the detection block, we have an interferogram $I(t)$,

$$
I(t)=A+B \cos \left[\frac{4 \pi}{\lambda} d(t)+\theta(t)\right]
$$

where $\lambda$ is the wavelength of the laser source, $d(t)$ is the displacement of the DUT and $\theta(t)$ is a random phase difference between the branches of the interferometer. If the displacement $d(t)$ is less than $\lambda / 2$, the detected intensity is

$$
I(t)=A+B\left[\cos \theta(t)-\frac{4 \pi}{\lambda} d(t) \sin \theta(t)\right]
$$

On high speed applications, such as in ultrasonics, $d(t)$ varies much faster than $\theta(t)$. Then we can write $I(t)$ as the sum of two terms,

$$
I(t)=I_{D C}+I_{A C}
$$

where,

$$
\begin{aligned}
I^{D C} & =A+B \cos \theta(t) \\
I^{A C} & =-B \frac{4 \pi}{\lambda} d(t) \sin \theta(t)
\end{aligned}
$$

The displacement $d(t)$ is encoded in the $I^{A C}$ component of the interferometric signal (Eq.(8)) and $\theta$ modulates both $I^{A C}$ and $I^{D C}$ components. The $I^{D C}$ component (Eq.(7)) limits the performance of the detector when measuring high speed signals by reducing the dynamic range of the sensor, as well as its effective bandwidth [1], [16], [17]. In summary, the interferogram consists of a small amplitude, high speed signal buried in a high amplitude, near-constant background intensity. Since $\theta(t)$ varies randomly, it is difficult to extract $d(t)$ when measuring small displacements [9]. In consequence, it is necessary to improve the ratio between $I^{A C}$ and $I^{D C}$. For this purpose, we designed the detection scheme showed in Fig.4.

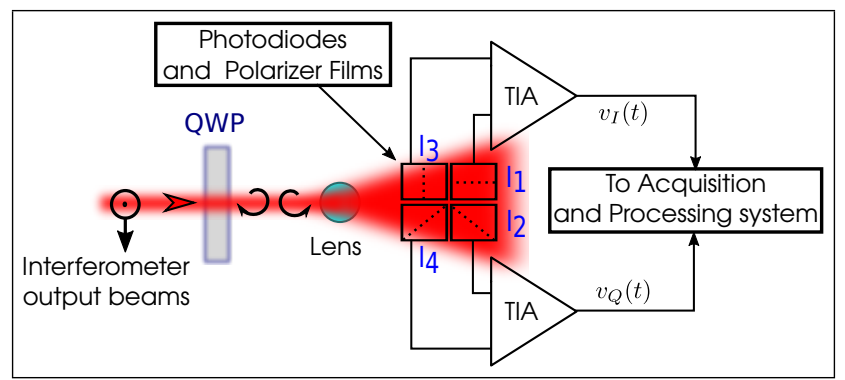

Fig. 4. Detection Block. The interferograms are produced by polarized films and detected with photodiodes. Dotted lines indicate the direction of the transmission axis of the films. The current provided by the photodiodes is converted into voltage signals $v_{I, Q}(t)$ by Transimpedance Amplifiers (TIAs). [6]

The detector produces four phase shifted interferograms (similar to the ones in Eq. (2)) that are converted into voltage signals by TIAs as we show in Fig. 4. The TIAs are made by operational amplifiers in differential mode. The output signals of the TIAs are voltage signals that depend on the difference in the currents of the photodiodes (i.e., the difference of the interferograms). The output signals $v_{I}$ and $v_{Q}$ are, respectively,

$$
\begin{aligned}
v_{I}(t) & =R_{\lambda}\left[I_{1}(t)-I_{3}(t)\right] R \\
v_{Q}(t) & =R_{\lambda}\left[I_{2}(t)-I_{4}(t)\right] R
\end{aligned}
$$

where the identical resistors $R$ determine the transimpedance gain of each TIA at the low frequency limit. The photodetectors (assumed identical) have responsivities $R_{\lambda}$. This way, the products $R_{\lambda} I_{N}(t)$ represent the currents produced by the photodiodes.

\section{A. Sensor implementation and characterization}

The sensor consists of four photodiodes BPW34s [18] and two FET-input operational amplifiers OPA657 [19] in transimpedance configuration. Its topology is shown in Fig. 5. The transimpedance amplifier was designed to achieve maximum transimpedance gain in a $10 \mathrm{MHz}$ bandwidth and to minimize the total input noise current $I_{E Q}$ (i.e. maximizing the SNR). $I_{E Q}$ is measured in $\mathrm{A} / \sqrt{\mathrm{Hz}}$ and represents the minimum input current, integrated over the bandwidth of the amplifier, which yielded a SNR of $0 \mathrm{~dB}$ 
up to the cutoff frequency. In order to achieve a maximally flat second order Butterworth frequency response, we must fulfil the following relation:

$$
\frac{1}{2 \pi R_{F} C_{F}}=\sqrt{\frac{G B P}{4 \pi R_{F} C_{D}}}
$$

where $R_{F}$ is the feedback resistance (transimpedance gain at low frequency) and $C_{F}$ is the feedback capacitance. Parallel to $R_{F}$ and $C_{D}$ is the total input capacitance at the inverter pin of the operational amplifier. This capacitance includes both the photodiode capacitance and the capacitance of the operational amplifier inputs. $G B P$ is the gain-bandwidth product of the amplifier. $R_{F}$ is calculated to achieve a bandwidth of $10 \mathrm{MHz}$ using,

$$
f_{-3 d B}=\sqrt{\frac{G B P}{2 \pi R_{F} C_{D}}}
$$

Instead of introducing an extra component, we take advantage of the parasitic capacitance of the resistor as the compensation capacitor $C_{F}$ to avoid instability issues. The total input noise current of the amplifier is,

$$
I_{E Q}=\sqrt{I_{N}^{2}+\left(\frac{4 k T}{R_{F}}\right)^{2}+\frac{\left(e_{N} 2 \pi C_{D} f_{-3 d B}\right)^{2}}{3}}
$$

where $I_{N}$ is the input current noise for the operational amplifier inverting input and $e_{N}$ is the input voltage noise. Full calculations and design considerations for Eqs. (11), (12), and (13) are found in [1], [14], [19], [20].

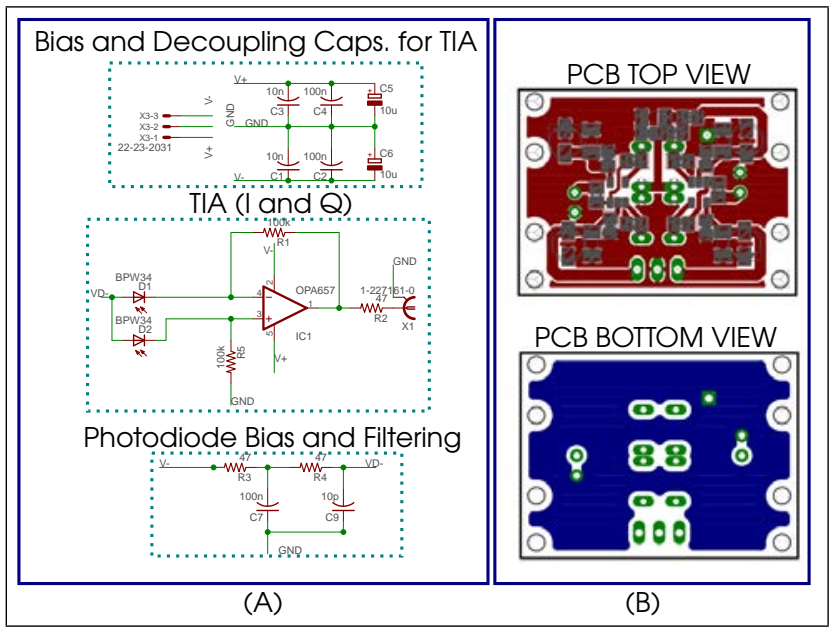

Fig. 5. Electrical implementation of the Quad Optical Sensor. (A) Schematic Diagram. (B) PCB Diagram. Red color is for top copper, blue is for bottom copper, vias are green, and the component mask is grey.

The photodiodes are very close to the inverter pin of the operational amplifiers and the decoupling capacitors are very close to their power supply. In this work, the transimpedance is set $R=100 \mathrm{k} \Omega$ to maximize the SNR in a bandwidth of $10 \mathrm{MHz}$. The printed circuit board (PCB) is etched on a FR4 substrate and has ground planes over both sides of the PCB to reduce electrical noise and interference through ground loops and to prevent crosstalk between adjacent circuit traces. The output paths of each TIA (one for photodiodes 1 and 3 and the other for photodiodes 2 and 4 ) are impedancematched to $50 \Omega$, have equal lengths, and are terminated with female BNC connectors. The voltage supply pins of each TIA are decoupled to ground by capacitors. The bias voltage of the photodiodes is decoupled by a second-order passive filter (cutoff frequency below $40 \mathrm{kHz}$ ). The size of the PCB is $40 \mathrm{~mm} \times 30 \mathrm{~mm}$ and the effective illuminated area (which includes the four photodiodes) is $7 \mathrm{~mm} \times 7$ $\mathrm{mm}$. The sensor developed is shown in Fig. 6 .

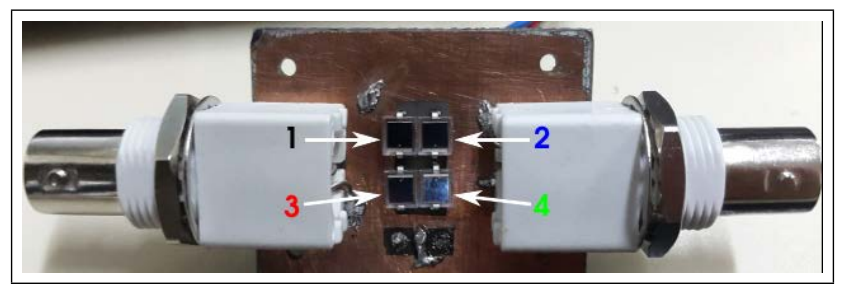

Fig. 6. Physical implementation of the Quad Optical Sensor. [6]

A separate test setup (Fig. 7) is used to characterize the electro-optical response of the sensor in the frequency range from $100 \mathrm{kHz}$ to $10 \mathrm{MHz}$. A LED light source with sinusoidal intensity modulation illuminates the four photodiodes uniformly. The modulation amplitude and linearity is verified independently using a ThorLabs PDA 155 optical detector [21].

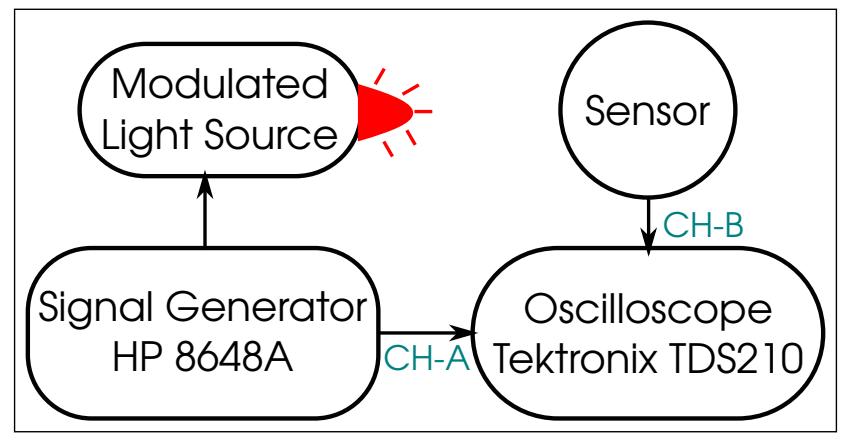

Fig. 7. Experimental scheme used for the characterization of the sensor. [6]

Each photodiode is characterized separately, covering the others with an opaque screen. At the low frequency limit $(100 \mathrm{kHz})$, the output signals from the four photodiodes have the same amplitude within the measurement uncertainty $( \pm 0.1 \mathrm{~dB})$. The frequency of the intensity modulation is swept linearly. The output signal is captured with an oscilloscope and then processed using a Fourier transformbased algorithm. A good match between the overall response of photodiodes and TIAs is achieved along the full frequency range, as shown in Fig. 8.

The relative amplitude response of the four photodiodes is shown in Fig. 8 (A). The $-3 \mathrm{~dB}$ frequency is approximately $8 \mathrm{MHz}$. There is also a good match of the phase response and group delay of the photodiodes and amplifiers, as shown in Fig. 8 (B) and Fig. $8(\mathrm{C})$. The phase response is approximately linear up to $3.7 \mathrm{MHz}$ with a slope of $0.5 \mu \mathrm{rad} / \mathrm{s}$. In summary, each photodetector and TIA has a flat relative amplitude and a linear phase response up to $3 \mathrm{MHz}$. These results show that we can use the sensor to analyze amplitude and phase responses of sub-nanometric DMI systems up to this frequency. In practice, if amplitude response is required 


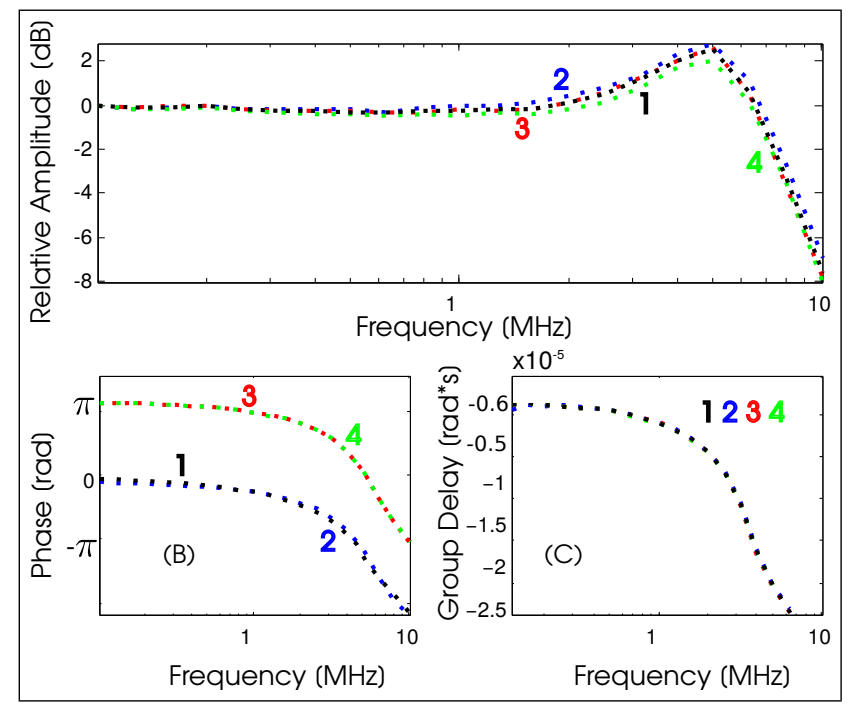

Fig. 8. Measured frequency response of the sensor. (A) Relative amplitude (B) Phase, (C) Group Delay. Measurement uncertainties are smaller than the thickness of the dotted lines. [6]

only, the frequency range may be extended up to $8 \mathrm{MHz}$. The measured response agrees with the design simulations.

The differential nature of the circuit (Fig. 5) reduces all $D C$ components and therefore increases the dynamic range of the amplifiers without compromising their gainbandwidth product [1]. We increase the common mode rejection ratio (CMRR) of the system by biasing both photodiodes to the same bias voltage $V_{\text {bias }}$. In this case, the impact on the performance of the detector due to a high $I^{D C}$ component is reduced, since we have a distributed photodetector configuration where each photodiode receives nearly a quarter of the total optical power delivered by the interferometer output. Since the outputs of the photodiodes are balanced, the output signals of the TIAs are practically insensitive to stray incoherent light and to intensity modulation of the laser source. This improves the overall robustness of the system and the signal to noise ratio (SNR) of the output signal of the TIAs [22].

In the next section, we analyze a design optimized for wideband applications, such as those found in heterodyne interferometry.

\section{THIRD APPROACH: WIDEBAND OPTICAL DETECTION}

An interferometric signal $I(t)$ is produced by the heterodyne polarization Michelson interferometer described in Fig. 9. The polarization plane of a linearly polarized $\mathrm{He}-\mathrm{Ne}$ laser is adjusted by means of a half-wave plate (HWP) to ensure that only the polarization normal to the plane of the experiment enters an acousto-optic modulator (AOM). The AOM is fed by an amplified radio-frequency (RF) generator which inputs a sinusoid with a frequency $f_{A O M}=70$ $\mathrm{MHz}$. The AOM is oriented at the Bragg angle to improve the diffraction efficiency to the first order. The first-order diffracted beam passes through a Polarization Beam Splitter (PBS) using a fixed mirror, and serves as the reference beam. The test beam is focused on the Device Under Test (DUT) with a microscope objective (MO), passing twice through a quarter waveplate (QWP) oriented at $45^{\circ}$ from the horizontal plane of the experiment, rotating the polarization plane of

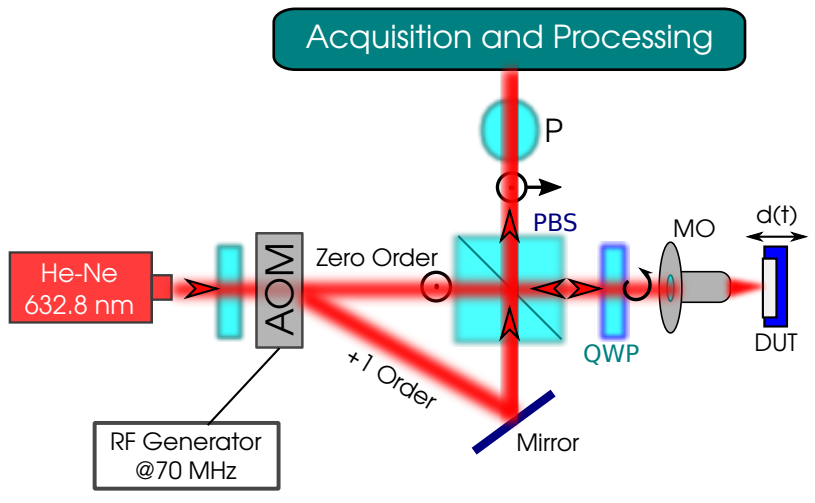

Fig. 9. A heterodyne Polarization Michelson interferometer. HWP: half waveplate, AOM: Acousto-Optic Modulator, PBS: polarized beam splitter, QWP: quarter waveplate, MO: microscope objective. P: Polarizer. $d(t)$ is the off-plane displacement of the device under test (DUT) to be measured.

the beam in order to reflect into the PBS, recombining with the reference beam. Using a polarizer $(\mathrm{P})$, we force the interference between the beams

$$
I(t)=A+B \cos \left[2 \pi f_{A O M} t+\frac{4 \pi}{\lambda} d(t)+\theta(t)\right]
$$

Heterodyne techniques offer the advantage of reducing the number of optical components and noise. The introduction of a carrier signal $f_{A O M}$ allows us to neglect the influence of $1 / f$ noise and reach the shot noise limit detection [23]. However, demodulation electronics are more complex and the bandwidth of the front ends are quite broader in comparison to the ones in homodyne systems.

\section{A. Front-end design and characterization}

An electrical implementation of the front-end is shown in Fig.10. The PCB was carefully designed to reduce parasitic capacitance in the sensitive parts of the circuit, such as the inverting pin of the operational amplifier and the feedback resistor.

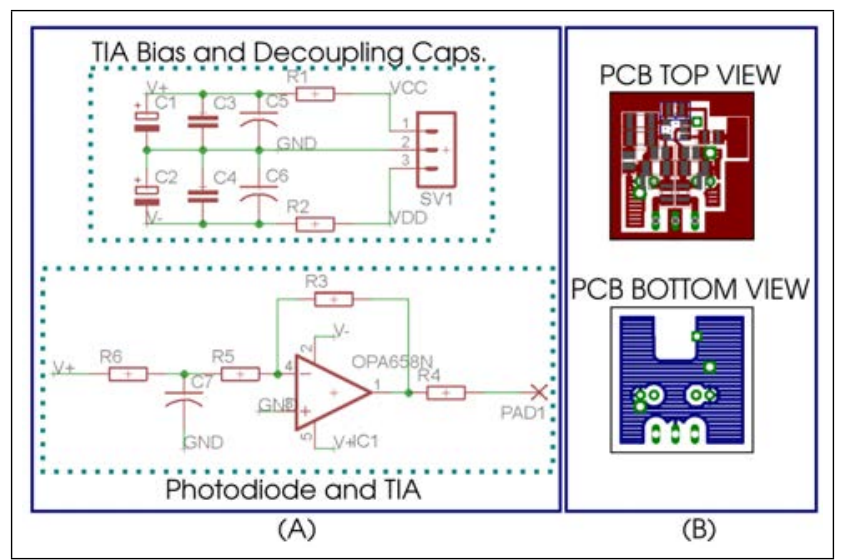

Fig. 10. Front end electrical implementation. (A) Schematic Design (B) PCB. Design. The ground plane at the bottom under the operational amplifier is removed to reduce parasitic capacitance in sensitive pins of the amplifier and feedback resistor.

To test the TIA, the photodiode (OPF482 OPTEK Technology [24]) was replaced by its Thevenin electrical representation: a highly valued resitor $R_{P H}$ and its junction 
capacitance $C_{P H}$ at the corresponding reverse voltage. The test setup is shown in Fig. 11 .

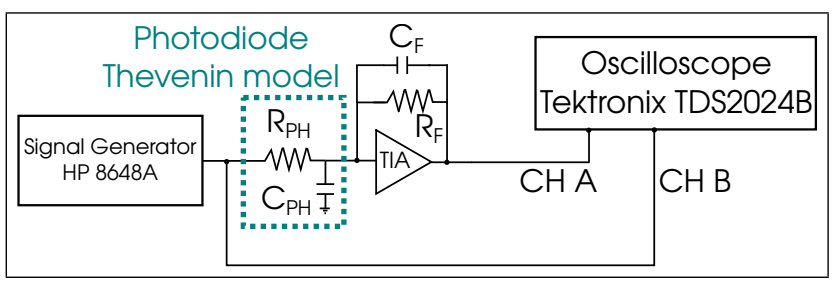

Fig. 11. Test setup for wideband TIA characterization. $R_{F}$ and $C_{F}$ are the feedback elements of the operational amplifier.

In Fig. 12 we show the measured transfer function of two implemented TIAs. One TIA uses the OPA657 operational amplifier from Texas Instruments [19] and the other one uses the LTC6268-10 from Linear Technology [25]. The photodiode parameters are $R_{P H}=22 \mathrm{k} \Omega$ and $C_{P H}=2$ $\mathrm{pF}$ in both cases.

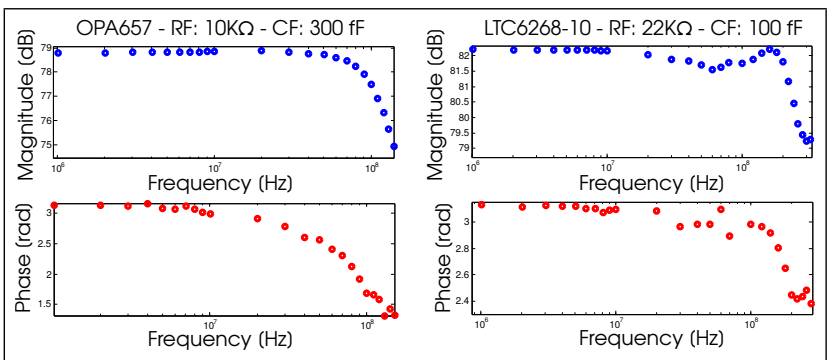

Fig. 12. Transfer function measurement of developed TIAs.

The measured $-3 \mathrm{~dB}$ frequency in the first TIA is 140 $\mathrm{MHz}$ and has a linear phase response up to $20 \mathrm{MHz}$. In the second case, the $-3 \mathrm{~dB}$ frequency is $320 \mathrm{MHz}$ approximately and has a linear phase response up to $40 \mathrm{MHz}$. The LTC6268-10 has a broader frequency response and has less noise than the OPA657. These characteristics arise from the fact that the LTC6268-10 has less input capacitance. However, the OPA657 has a flatter magnitude response. The peak observed around $160 \mathrm{MHz}$ in the transfer function of the LTC6268-10 is also seen in [25]. An implementation of the photodiode TIA using the OPA657 is shown on Fig.13.

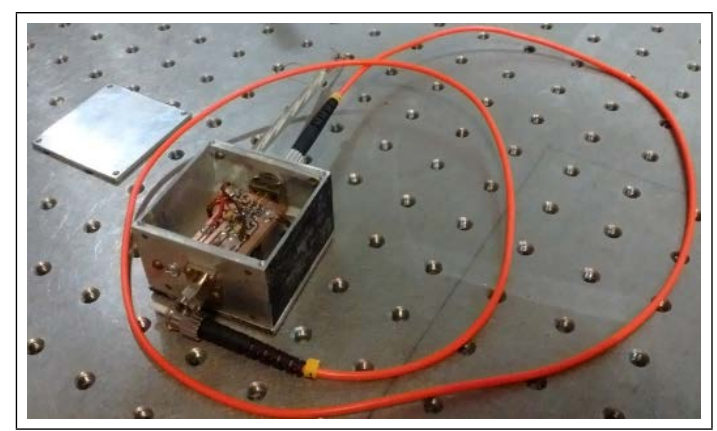

Fig. 13. Implementation of a photodiode transimpedance amplifier using an OPA657 operational amplifier.

A metal enclosure isolates the circuit from external noise sources. The photodiode is coupled to a multimode fiber by an ST connector. The output connector of the TIA is a $50 \Omega$ male SMA connector.

\section{CONCLUSIONS}

We described the design and performance of three transimpedance amplifiers optimized for optical dynamic interferometry. In the first case, the most significant characeteristics of the detector arise from the photodiode parameters. In the second case, we present the typical topology of the operational amplifier TIA, for medium frequency applications. Moreover, we present an approach to balanced detection to improve the quality of the measurement and the resolution of the entire interferometric scheme. Finally, we present a wideband TIA implementation for heterodyne optoelectronics. The design considerations are similar to the second case: the gain and bandwidth must be set to maximize the SNR in the band of interest, but good practices in high speed PCB layout must be applied to overcome parasitics. As a prospective, we will analyze the design of ultra high speed transimpedance amplifiers for optical pulse measurements.

\section{ACKNOWLEDGEMENTS}

This work is supported by four UBACYT grants from Universidad de Buenos Aires (20142017 UBACYT 20020130100346BA, UBACYT 2016-2017 20020150200143BA, UBACYT 20172019 20020160100042BA, UBACYT 2017-2019 20020160100052BA). The corresponding author acknowledges a doctoral scholarship from CONICET.

\section{REFERENCES}

[1] J. Graeme, Photodiode Amplifiers: Op Amp Solutions, ser. Gain technology. McGraw Hill, 1996. [Online]. Available: https://books.google.co.uk/books?id=sHV0c5hBW4QC

[2] D. Guo, M. Wang, and H. Hao, "Displacement measurement using a laser feedback grating interferometer," Applied optics, vol. 54, no. 31, pp. 9320-9325, 2015.

[3] N. E. Myridis, "Fundamentals of picoscience, edited by klaus d. sattler," Contemporary Physics, vol. 56, no. 1, pp. 95-95, 2015. [Online]. Available: http://dx.doi.org/10.1080/00107514.2014.962096

[4] L. Miccio, P. Memmolo, F. Merola, S. Fusco, V. Embrione, A. Paciello, M. Ventre, P. A. Netti, and P. Ferraro, "Particle tracking by full-field complex wavefront subtraction in digital holography microscopy," Lab Chip, vol. 14, pp. 1129-1134, 2014. [Online]. Available: http://dx.doi.org/10.1039/C3LC51104A

[5] A. Rosenthal, S. Kellnberger, D. Bozhko, A. Chekkoury, M. Omar, D. Razansky, and V. Ntziachristos, "Sensitive interferometric detection of ultrasound for minimally invasive clinical imaging applications," Laser \& Photonics Reviews, vol. 8, no. 3, pp. 450-457, 2014.

[6] L. M. Riobo, F. E. Veiras, P. A. Sorichetti, and M. T. Garea, "Wideband quad optical sensor for high-speed sub-nanometer interferometry," Appl. Opt., vol. 56, no. 3, pp. 397-403, Jan 2017. [Online]. Available: http://ao.osa.org/abstract.cfm?URI=ao-56-3-397

[7] S. Kurmulis and L. Novotny, "Common path heterodyne interferometer for single nanoparticle detection in fluids," in Frontiers in Optics 2016. Optical Society of America, 2016, p. FTu2F.5. [Online]. Available: http://www.osapublishing.org/abstract.cfm?URI=FiO-2016-FTu2F.5

[8] B. P. Abbott et al., "Observation of gravitational waves from a binary black hole merger," Phys. Rev. Lett., vol. 116, p. 061102, Feb 2016. [Online]. Available: https://link.aps.org/doi/10.1103/PhysRevLett.116.061102

[9] N. Alvarez, M. T. Garea, and F. P. Quintián, "Ultrasonic lamb waves detection using an unstabilized optical interferometer with uncalibrated phase shifts," Journal of Modern Optics, vol. 62, no. 7, pp. 556-559, 2015

[10] P. K. Rastogi, Digital Speckle Pattern Interferometry \& Related Techniques. New York, NY, USA: John Wiley \& Sons, Inc., 2000.

[11] G. P. Yu Fu and X. Li, "Interferometric dynamic measurement: Techniques based on high-speed imaging or a single photodetector," The Scientific World Journal, vol. 2014, pp. 1-14, 2014. [Online]. Available: https://www.hindawi.com/journals/tswj/2014/232906/cta/ 
[12] F. E. Veiras, L. M. Riobó, C. L. Matteo, L. I. Perez, and M. T. Garea, "Birefringent phase demodulator: application to wave plate characterization," Appl. Opt., vol. 54, no. 9, pp. 2326-2333, Mar 2015. [Online]. Available: http://ao.osa.org/abstract.cfm?URI=ao-549-2326

[13] L. M. Riobó, Dispositivos demoduladores de fase de senales ópticas con cristales birrefringentes. Facultad de Ingeniería, Universidad de Buenos Aires, CABA, Sep 2014, Tesis de grado Ingeniería Electrónica.

[14] M. Johnson, Photodetection and Measurement: Making Effective Optical Measurements for an Acceptable Cost, ser. Professional Engineering. McGraw-Hill Education, 2003. [Online]. Available: https://books.google.com.ar/books?id=K5IKWDwUYZAC

[15] Vishay Semiconductors, "BPW43," https://www.vishay.com/docs/81523/81523.pdf, Silicon PIN Photodiode.

[16] P. S. Matavulj, D. M. Gvozdić, J. B. Radunović, and J. M. Elazar, "Nonlinear pulse response of p-i-n photodiode caused by the change of the bias voltage," International Journal of Infrared and Millimeter Waves, vol. 17, no. 9, pp. 1519-1528, 1996. [Online]. Available: http://dx.doi.org/10.1007/BF02088505

[17] M. Dentan and B. de Cremoux, "Numerical simulation of the nonlinear response of a p-i-n photodiode under high illumination," Journal of Lightwave Technology, vol. 8, no. 8, pp. 1137-1144, Aug 1990.

[18] Vishay Semiconductors, "BPW34, BPW34s," http://www.vishay.com/docs/81521/bpw34.pdf, silicon PIN Photodiode.

[19] Texas Instruments, "OPA657," http://www.ti.com/lit/ds/symlink/opa657.pdf, OPA657 1.6-GHz, Low-Noise, FET-Input Operational Amplifier.

[20] P. Staric and E. Margan, Wideband amplifiers. Springer, 2006.

[21] Thorlabs Inc., "PDA155 - Amplified Si Photodetector," https://www.thorlabs.de/drawings/6fd7b2edbf13b66c-3F32185B5056-2306-D9F732FD98FB4D18/PDA155-Manual.pdf, pDA155 Amplified Si Photodetector.

[22] C. Scruby and L. Drain, Laser Ultrasonics Techniques and Applications. Taylor \& Francis, 1990. [Online]. Available: https://books.google.com.ar/books?id=KgXPqx9ST- $w C$

[23] E. Leirset, H. E. Engan, and A. Aksnes, "Heterodyne interferometer for absolute amplitude vibration measurements with femtometer sensitivity," Opt. Express, vol. 21, no. 17, pp. 19900-19921, Aug 2013. [Online]. Available: http://www.opticsexpress.org/abstract.cfm?URI=oe-21-17-19900

[24] OPTEK Technology, "OPF482," http://uk.farnell.com/optektechnology/opf482/receiver-fibre-optic-to-18-st/dp/1497962, OPF482 Fiber Optic Detector.

[25] Linear Technology,

"LTC6268-10," http://www.linear.com/product/LTC6268-10， LTC6268-10 - 4GHz Ultra-Low Bias Current FET Input Op Amp. 